\title{
A tight lower bound for convexly independent subsets of the Minkowski sums of planar point sets*
}

\author{
Ondřej Bílka ${ }^{\dagger}$ Kevin Buchin ${ }^{\ddagger}$ Radoslav Fulek ${ }^{\S}$ Masashi Kiyomi \\ Yoshio Okamotoll Shin-ichi Tanigawa* ${ }^{* *}$ Csaba D. Tóth ${ }^{\dagger \dagger}$
}

Submitted: Jul 31, 2009; Accepted: Oct 13, 2010; Published: Oct 29, 2010

Mathematics Subject Classification: 52C35, 52A10

\begin{abstract}
Recently, Eisenbrand, Pach, Rothvoß, and Sopher studied the function $M(m, n)$, which is the largest cardinality of a convexly independent subset of the Minkowski sum of some planar point sets $P$ and $Q$ with $|P|=m$ and $|Q|=n$. They proved that $M(m, n)=O\left(m^{2 / 3} n^{2 / 3}+m+n\right)$, and asked whether a superlinear lower bound exists for $M(n, n)$. In this note, we show that their upper bound is the best possible apart from constant factors.
\end{abstract}

\section{Introduction}

Recently, Eisenbrand, Pach, Rothvoß, and Sopher [1] studied the function $M(m, n)$, which is the largest cardinality of a convexly independent subset of the Minkowski sum of some planar point sets $P$ and $Q$ with $|P|=m$ and $|Q|=n$. They proved that $M(m, n)=$

*Preliminary versions of our results have been presented at the Czech-Slovakian Student Competition in Mathematics and Computer Science (Kosice, May 27-29, 2009), and at the 7th Japan Conference on Computational Geometry and Graphs (Kanazawa, November 11-13, 2009). A part of the work has been done at the 12th Korean Workshop on Computational Geometry, June 2009 and at the 7th Gremo Workshop on Open Problems, July 2009. The authors thank the organizers of these workshops.

${ }^{\dagger}$ Charles University. Email: ondra@kam.mff.cuni.cz.

†Technische Universiteit Eindhoven. Email: k.a.buchin@tue.nl. Supported by the Netherlands’ Organisation for Scientific Research (NWO) under project no. 639.022.707.

§École Polytechnique Fédérale de Lausanne. Email: radoslav.fulek@epfl.ch. Partially supported by "Discrete and Convex Geometry project (MTKD-CT-2005-014333) of the European Community."

IJapan Advanced Institute of Science and Technology. Email: mkiyomi@jaist.ac.jp.

${ }^{\|}$Tokyo Institute of Technology. Email: okamoto@is.titech.ac.jp. Supported by Global COE Program "Computationism as a Foundation for the Sciences" and Grant-in-Aid for Scientific Research from Ministry of Education, Science and Culture, Japan, and Japan Society for the Promotion of Science.

**Kyoto University. Email: tanigawa@kurims.kyoto-u.ac.jp.

††University of Calgary. Email: cdtoth@math.ucalgary.ca. Supported in part by NSERC grant RGPIN 35586. 


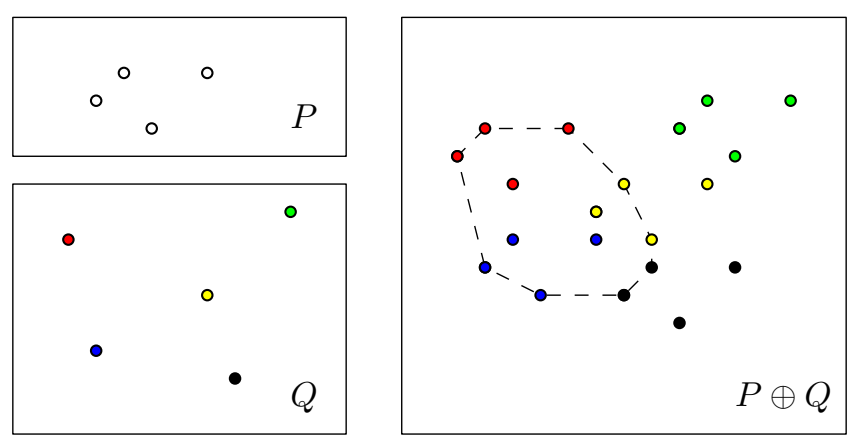

Figure 1: An example.

$O\left(m^{2 / 3} n^{2 / 3}+m+n\right)$, and asked whether a superlinear lower bound exists for $M(n, n)$. The quantity $M(n, n)$ gives an upper bound for the largest convexly independent subset of $P \oplus P$, and it is related to the convex dimension of graphs, proposed by Halman, Onn, and Rothblum [3]. Figure 1 shows an example. In this note, we show that the upper bound presented in [1] is the best possible apart from constant factors.

Theorem 1. For every $m, n \in \mathbb{N}$, there exist point sets $P, Q \subset \mathbb{R}^{2}$ with $|P|=m,|Q|=$ $n$ such that the Minkowski sum $P \oplus Q$ contains a convexly independent subset of size $\Omega\left(m^{2 / 3} n^{2 / 3}+m+n\right)$.

\section{Definitions}

The Minkowski sum of two sets $P, Q \subseteq \mathbb{R}^{d}$ is defined as $P \oplus Q=\{p+q \mid p \in P, q \in Q\}$. A point set $P \subseteq \mathbb{R}^{d}$ is convexly independent if every point in $P$ is an extreme point of the convex hull of $P$.

\section{Basic idea}

Let $n$ and $m$ be integers. Let $P$ be a planar point set that maximizes the number of point-line incidences between $m$ points and $n$ lines. Erdös [2] showed that for $m, n \in \mathbb{N}$, there exist a set $P$ of $m$ points and a set $L$ of $n$ lines in the plane with $\Omega\left(m^{2 / 3} n^{2 / 3}+m+n\right)$ point-line incidences. A point-line incidence is a pair of a point $p$ and a line $\ell$ such that $p \in \ell$ (that is, $p$ lies on $\ell$ ). Szemerédi and Trotter [6] proved that this bound is the best possible, confirming Erdős' conjecture (see [4] for the currently known best constant coefficients).

Sort the lines in $L$ by the increasing order of their slopes (break ties arbitrarily). Denote by $P_{i}$ the set of points in $P$ that are incident to the $i$ th line in $L$. Consider a polygonal chain $C$ consisting of $|L|$ line segments such that the $i$ th segment $s_{i}$ has the same slope as the $i$ th line of $L$. Since we sorted the lines in $L$ by their slopes, $C$ is a (weakly) convex chain. Set the length of each line segment to be at least the diameter of the point set $P$. The chain $C$ has $n+1$ vertices including two endpoints. Now we can 


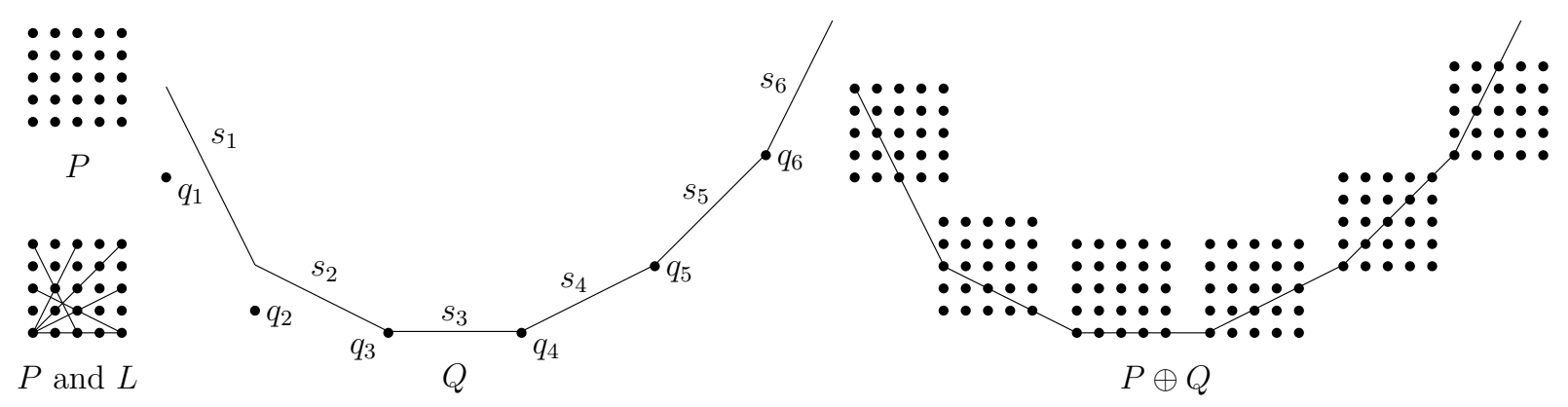

Figure 2: Basic idea for our construction.

describe our point set $Q=\left\{q_{1}, \ldots, q_{n}\right\}$. The $i$ th point $q_{i}$ is placed on the plane so that the points in $P_{i} \oplus\left\{q_{i}\right\}$ all lie on $s_{i}$. This concludes the construction of $Q$. See Figure 2 for an illustration.

The number of points in $P \oplus Q$ that lie on $C$ is $\Omega\left(m^{2 / 3} n^{2 / 3}+m+n\right)$ since if $p \in P_{i}$ then $p+q_{i} \in s_{i} \subseteq C$. Thus in the above construction, $(P \oplus Q) \cap C$ is a subset of $P \oplus Q$ that contains $\Omega\left(m^{2 / 3} n^{2 / 3}+m+n\right)$ points in (weakly) convex position.

\section{$4 \quad$ Fine tuning}

The point set $(P \oplus Q) \cap C$ is not necessarily convexly independent for two reasons:

1. Some of the lines in $L$ may be parallel.

2. For each $i$, the points in $(P \oplus Q) \cap s_{i}$ are collinear.

We next describe how to overcome these issues.

For the first issue, we apply a projective transformation to $P$ and $L$ (see e.g. [5]). A generic projective transformation maps $P$ to a set of real points, and $L$ to a set of pairwise nonparallel lines. Since projective transformations preserve incidences, the number of incidences remains $\Omega\left(m^{2 / 3} n^{2 / 3}+m+n\right)$. By applying a rotation, if necessary, we may assume that no line in $L$ is vertical. Therefore, without loss of generality we may assume that all lines of $L$ have different non-infinite slopes. As before we sort the lines in $L$ in the increasing order by their slopes.

For the second issue, we apply the following transform to $P$ and $L$ (after the projective transformation and the rotation above): Each point $(x, y)$ in the plane is mapped to $\left(x, y+\varepsilon x^{2}\right)$ for a sufficiently small positive real number $\varepsilon$. Then the $i$ th line $y=a_{i} x+b_{i}$ is mapped to the convex parabola $y=\varepsilon x^{2}+a_{i} x+b_{i}$. By scaling the whole configuration, we may assume that the $x$-coordinates of all points of $P$ are properly between 0 and 1 . Then, the gradient of the $i$ th parabola is $a_{i}$ at $x=0$ and $a_{i}+2 \varepsilon$ at $x=1$. Let $\varepsilon$ be so small that the intervals $\left[a_{i}, a_{i}+2 \varepsilon\right]$ are all disjoint: Namely, the gradient of the $i$ th parabola at $x=1$ is smaller than the gradient of the $(i+1)$ st parabola at $x=0$ (or more specifically it is enough to choose $\left.\varepsilon=\min \left\{\left(a_{i}-a_{i-1}\right) / 3 \mid i=2, \ldots, n\right\}\right)$. Therefore, instead of constructing a convex chain by line segments, we construct a convex chain $C$ consisting 
of convex parabolic segments: The $i$ th segment is a part of an expanded copy of the $i$ th parabola (containing the piece between $x=0$ and $x=1$ ). From the discussion above, these parabolic segments together form a strictly convex chain and we can construct the point set $Q$ in the same way as the previous case. Thus, for these $P$ and $Q$, the set $(P \oplus Q) \cap C$ is a convexly independent subset in $P \oplus Q$ of size $\Omega\left(m^{3 / 2} n^{3 / 2}+m+n\right)$. Q.E.D.

\section{An open problem}

Let $M_{k}(n)$ denote the maximum convexly independent subset of the Minkowski sum $\bigoplus_{i=1}^{k} P_{i}$ of $k$ sets $P_{1}, P_{2}, \ldots, P_{k} \subset \mathbb{R}^{2}$, each of size $n$. Our lower bound in the case $m=n$, combined with the upper bound in [1] shows that $M_{2}(n)=\Theta\left(n^{4 / 3}\right)$. Determine $M_{k}(n)$ for $k \geqslant 3$.

\section{References}

[1] F. Eisenbrand, J. Pach, T. Rothvoß, and N. B. Sopher. Convexly independent subsets of the Minkowski sum of planar point sets. The Electronic Journal of Combinatorics 15 (2008), N8.

[2] P. Erdős. On a set of distances of $n$ points. The American Mathematical Monthly 53 (1946) 248-250.

[3] N. Halman, S. Onn, and U. G. Rothblum. The convex dimension of a graph. Discrete Applied Mathematics 155 (2007) 1373-1383.

[4] J. Pach, R. Radoicic, G. Tardos, and G. Tóth. Improving the crossing lemma by finding more crossings in sparse graphs. Discrete and Computational Geometry 36:4 (2006) 527-552.

[5] F. P. Preparata and M. I. Shamos. Computational Geometry: An Introduction. Springer Verlag, New York, 1985.

[6] E. Szemerédi and W. Trotter, Jr. Extremal problems in discrete geometry. Combinatorica 3 (1983) 381-E92. 